\title{
MEASUREMENT ERRORS AND UNCERTAINTY ESTIMATION OF AN EXPERIMENTAL SET UP USING A 2D PIV TECHNIQUE
}

\author{
Flávia V. Barbosa \\ MEtRICs I\&D Centre \\ School of Engineering \\ University of Minho, \\ Guimarães, Portugal \\ Senhorinha F. C. F. Teixeira \\ ALGORITMI I\&D Centre \\ School of Engineering \\ University of Minho, Guimarães, Portugal
}

\author{
Carlos A. P. Costa \\ MEtRICs I\&D Centre \\ School of Engineering \\ University of Minho, \\ Guimarães, Portugal \\ José C. F. Teixeira \\ MEtRICs I\&D Centre \\ School of Engineering \\ University of Minho, Guimarães, Portugal
}

\section{ABSTRACT}

The study of the flow interaction and the heat transfer between air jets and a surface is of paramount importance in industrial processes that apply multiple air jet impingement. To ensure a good performance of the process, high heat transfer rates and uniformization of the flow over the target plate are required. To perform this analysis, a PIV technique was implemented for the measurement of the velocity fields of the flow. However, as any real experiment, the values recorded by the PIV method are subjected to several errors that compromise the reliability and accuracy of the measurements. These errors can have different sources, from the installation and alignment to the particles seeding and calibration procedure. To determine an interval that contains the measurement error, the uncertainty quantification is crucial. In that sense, this paper focus on the identification of measurement errors and uncertainty quantification of an experimental set up specially built for the analysis of the interaction between a non-isothermal jets and non-flat surfaces moving perpendicularly to the jet axis. To ensure the reliability of the results, preliminary tests were performed to guarantee a uniform and stable flow and to determine the range and conditions of operation. In addition, this work presents an analysis of the system, and the source of errors are identified, quantified and, when possible, corrected. The particle seeding, which consists of olive oil droplets, is characterized and its efficiency for the flow tracking is analysed. The test facility was tested to fully characterize the flow field in terms of mean velocity profile and turbulence intensity over a wide range of Reynolds numbers and temperature. Several velocity fields are then measured until convergence of the flow quantities is reached. The combination of these measurements with high spatial resolution and low measurement errors allow to obtain accurate and precise measurement values.

Keywords: Jet impingement; PIV; Uncertainty; Experimentation; Numerical Simulation.

\section{INTRODUCTION}

Jet impingement is a complex heat transfer process that involves flow interactions between the jet and the target surface.

In several industrial processes such as heat treatment, drying, food processing, reflow soldering, cooling of turbine and electronic components [1], high average heat transfer coefficients and the uniformity of the heat transfer over the impinging surface are required to improve the performance of the process and to avoid local hot (or cold) spots. These applications require large areas, and therefore, a single jet system is not efficient, being the multi-jet configuration more appropriate. Although this process allows the maximum heat transfer rates among all the single phase heat transfer methods [2], its performance is influenced by several geometrical and flow parameters such as Reynolds number, Prandtl number and Mach number, jet-to-jet spacing, radial distance from stagnation point, target plate inclination, confinement of the jet, nozzle 
geometry, curvature of the target plate, roughness of the target plate and turbulence intensity at the nozzle exit [3].

Due to the complexity and large industrial applicability of the jet impingement process, its study is highly relevant in order to enhance the convective heat transfer process, but also to improve the heat transfer uniformity and the coverage of the impinging surface. To perform a study of the multiple jet impingement process, several techniques have been conducted. Angioletti et al. [4] characterized the flow field and local heat transfer of jet impingement applying a particle image velocimetry (PIV) technique. They estimated an overall error according to [5] [6] of about $4 \%$ on velocity average. The authors observed an axial velocity pulsation that leads to a decrease of the boundary layer stability at the stagnation point which affects the local heat transfer.

Ichikawa et al. [7] investigated the flow behavior of an array of circular jets by changing the nozzle-to-plate $(2 D$ to $6 D)$ and the jet-to-jet spacing $(4 D$ to $6 D)$ at $\mathrm{Re}=3000$. The authors used the stereoscopic Particle Image Velocimetry (PIV) technique to measure the flow field and the Thermosensitive Liquid Crystal (TLC) method to obtain the heat transfer coefficients. The measured data allow to observe that the heat transfer magnitude and distribution is associated to the jet co-axial velocity component close to the wall.

Xing et al. [8] analyzed the effects of the Reynolds number, crossflow, jets patterns and jet-to-plate spacing, on the distribution of the local Nusselt number. To conduct this study, they applied the Monochromic Liquid Crystal for the measurement of the heat transfer, being the local heat transfer coefficient determined using the measured wall temperature and the dimensional transient heat conduction in a semi-infinite solid. For the measurement uncertainties [8] used the Kline and McClintock [9] approach based on the accuracy of the thermocouples, the calibration of the Monochromic Liquid Crystal and the time detection.

Li et al. [10] studied the influence of the Reynolds number, jet-to-jet spacing, je-to-target distance and jet inclination on the local and average heat transfer coefficients under an inline impinging jet array at 5,000 $\leq \mathrm{Re} \leq 25,000$. To measure the heat transfer coefficients, they used a Transient Liquid Crystal technique. The authors conducted an uncertainty analysis, based on Moffat [11], considering the errors associated to the measurement of the heat transfer coefficients, the temperatures over the target surface and the time detection.

Grenson et al. [12] investigated a hot air jet impinging a flat plate at $\operatorname{Re}=60,000$ and $H / D=3$. The description of the flow field was performed using a stereoscopic particle image velocimetry (S-PIV) and Laser Doppler Velocimetry (LDV) methods while the heat transfer distribution over the plate was measured through infrared thermography. The authors observed that PIV fails to provide accurate measurements in the near wall jet region due essentially to the light reflection that alters the signal to noise ratio and the limited spatial resolution caused by the experimental setup which difficult the acquisition of images that capture strong flow variations. An underestimation of axial velocity fluctuation is also observed due to the lower seeding density observed far from the jet axis.

Terzis [13] studied the flow field and heat transfer in a multiple jet configuration exposed in a self-gained crossflow using a PIV technique and a liquid crystal thermography (LCT). The authors identified the complex flow structure of the jet impingement and observed two maximum heat transfer values, one at the stagnation point and the other one in a region were both velocity components are decelerated.

Kalifa et al. [14] analyzed the flow and heat transfer of a round air jet impinging an heated plate considering a Reynolds number of $1,000,2,000$ and $3,000, H / D=1$ and 2 using a PIV and LDV techniques. The authors observed that the increase of the Reynolds number leads to instability on the wall jet and an approximation of the separation point to the jet axis with the increase of $H / D$. In addition, the increase of the plate temperature leads to the decrease of the vertical velocity, being the separation point identified closer to the jet axis.

Through the above-mentioned literature, it is clear that the jet impingement process is still an area of interest and several techniques are applied to conduct an accurate study in order to identify the complex interactions between the jet and the target plate. However, the identification of the source of errors related to the experimental study of the jet impingement process is slightly approached in literature. Considering the relevance of this subject for the correct characterization of the experimental setup and increase of the level of confidence of the results obtained in PIV measurements, it is approached in this work. This paper presents the source of errors identified in a speciallybuilt experimental setup for the study of multiple jets impinging a target surface and approaches the influence of these factors in the reliability and accuracy of the measurement obtained by PIV technique. With this study, it is intended to reduce all the errors that are related with the experimental apparatus, increasing the level of confidence of the results obtained. It is given special attention to this subject since the results obtained experimentally will be the base for the construction of a numerical model that simulates an industrial process based on multiple jet impingement, the reflow soldering. In that sense, the level of confidence of the results obtained must be high to ensure that the computational model predicts with accuracy the physical phenomena.

\section{EXPERIMENTAL APPARTUS}

\section{Experimental setup}

The experimental setup was projected based on an industrial process for the production of Printed Circuit Boards (PCB), the reflow soldering. This process consists of an oven with multiple air jets that heats and cool the impinging surface in function of the thermal profile previously defined. The PCB inside the oven pass first through a heating process to melt the solder paste and wet the joints, followed by a cooling process to connect the electronic component to the PCB. However, several defects 
arise from this process and a rigorous study must be conducted in order to reduce the productivity loss.

Thus, this study focuses on the forced convection by multiple jet impingement in the heating zone. An experimental setup was designed and constructed with the aim to heat a target plate with different configurations at a specific temperature and through multiple air jets. The configuration of the oven is presented in the schematic of Fig. 1 . The hot air $\left(120^{\circ} \mathrm{C} / 150^{\circ} \mathrm{C}\right)$ is generated by a high temperature centrifugal ventilator with a power of $900 \mathrm{~W}$ and a maximum flow rate of $565 \mathrm{~m}^{3} / \mathrm{h}(1)$ before passing through a divergent section (2), which reduce the pressure drop, a honeycomb structure (3) for the uniformization of the flow and a stabilization zone (4). These components reduce the turbulence of the flow, ensuring a uniform distribution of the air that flows through the circular nozzles (5), generating hot air jets. These multiple jets impinge a target plate located at the test chamber (6). To ensure the measurement of the velocity field through a PIV system, the test chamber is equipped by a window and a glass door perpendicularly positioned. The air is heated by heaters located at (7). Since it is a closed-loop system, the air returns to the fan through a conduct (8).

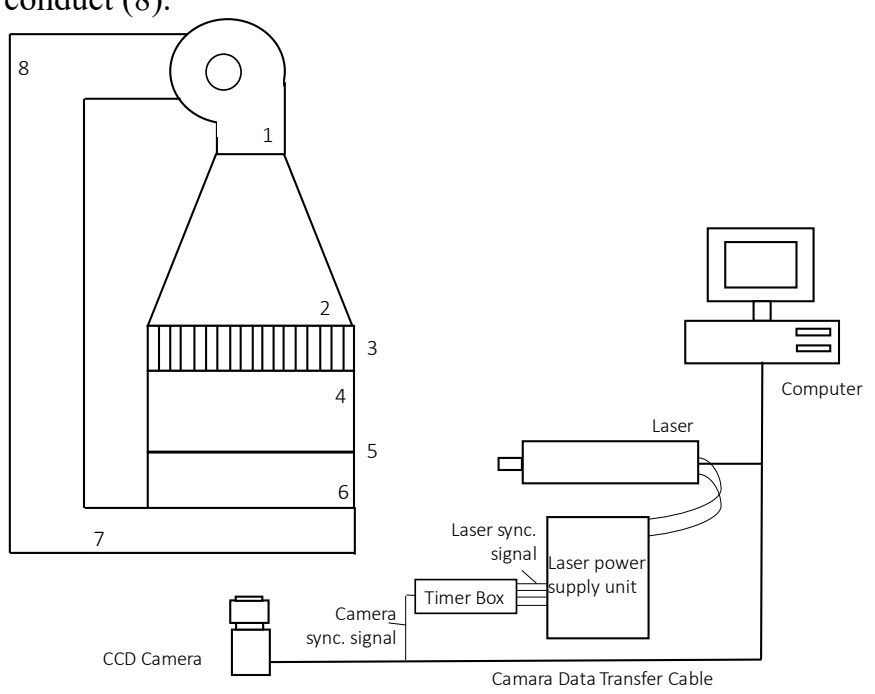

Fig. 1 - Schematic of the experimental setup.

\section{PIV technique}

As mentioned, a PIV system was employed to analyze the velocity of the jet impingement. This technique consists on the detection of the particle motion at a finite observation time $\Delta t$ [15]. The system, illustrated schematically in Fig. 1, consists on a $145 \mathrm{~mJ}$ double-pulse Nd:YAG laser firing on the second harmonic, i.e. green $532 \mathrm{~nm}$. A two dimensional laser sheet of is obtained by the two beams previously recombined on the same optical path by a polarized dichroic filter and expanded in one direction through a combination of spherical and cylindrical lens [4]. This laser sheet illuminates the measurement region from the exit of the nozzles to the target plate over all the length of the test chamber.
The velocity field measurement is ensured by tracer particles introduced inside the system using a smoke generator. These particles aim to follow the instantaneous motion of the air and scatter the light. The characterization of the seeding is presented in the following chapter. To capture the images, a HiSense Zyla CCD camera is positioned at an angle of $90^{\circ}$ with the laser. This camera has a pixel size of $6.5 \mu \mathrm{m}$ and a pixel resolution of $2560 \times 2160$ (5.5 Megapixel). An electronic synchronizer ensures the synchronization between the CCD camera, the two laser beams and the frame grabber. The CCD camera captures two consecutive images spaced by a short time interval in order to visualize the displacement of the tracking particles. The data acquisition and processing of the images are performed by the software Dynamic Studio. The images obtained are divided into interrogation areas and mathematical correlation are applied to obtain velocity vectors [16].

Although the PIV methods is highly efficient for the measurement of velocity fields, the performance and accuracy of this technique depends on many parameters. To ensure the reliability of the results obtained by PIV measurements, it is important to identify these parameters and to determine the source of errors that can decrease the efficiency and quality of the measurements. This analysis is conducted in the following section.

\section{SOURCE OF ERRORS RELATED TO THE EXPERIMENTAL APPARTUS}

\section{Uncertainty and Measurement Error}

In PIV technique, the velocity field of the flow is obtained indirectly as a displacement of the tracer particles in a finite time interval as presented in Eq.1 [17]:

$$
D\left(X ; t^{\prime}, t^{\prime \prime}\right)=\int_{t^{\prime}}^{t^{\prime \prime}} v[X(t), t] d t
$$

where, $D\left(X ; t^{\prime}, t^{\prime \prime}\right)$ is the tracer particle displacement and $v[X(t), t]$ its velocity. For ideal particles, $v$ must be equal to the velocity of the flow in study. The PIV system will analyze this displacement by image analysis, and so, the instantaneous velocity given by the system is such as Eq. (2):

$$
V_{p}=S \frac{\Delta x_{p}}{\Delta t}
$$

where $S$ is the scaling factor, $\Delta x_{p}$ is the displacement in pixels and $\Delta t$ the time between the two consecutive images recorded by the camera [18]. In that sense, the basic formula to represent the uncertainty linked to the velocity measurement can be expressed as Eq. (3) [18]:

$$
U_{\% V_{p}}=\sqrt{\left(u_{\% s}\right)^{2}+\left(u_{\% \Delta x_{p}}\right)^{2}+\left(u_{\Delta t}\right)^{2}}
$$


The magnitude of these uncertainties is directly related to the source of errors related to the measurement system, which include the installation, the PIV system and the conditions of operation of the experiments.

These measurement errors are typically divided in random and systematic errors. The first ones are characterized by its non-predictable nature, since they can change in magnitude and sign for each measurement. In contrast, systematic errors are predictable if known and constant by nature, in this way, they can be compensated if identified before the measurement, being the accuracy of the results obtained determined by their magnitude [19]. These errors can be originated by incorrect operation of the measurement system or inappropriate calibration. Since systematic errors are inherent to the experimental apparatus, they will be approached in this paper.

\section{PIV error sources}

The extensive research and continuous improvement of the PIV system have made it an established and reliable tool for the measurement of velocity fields both in 2D and 3D flows [20]. This important measurement tool has been used to support the development of numerical models of complex flows and validate the simulations [21]. In that sense, the reliability of the results measured by the PIV are crucial to ensure the numerical predictions.

The accuracy of the measurements depends on several factors from the flow under study to the experimental setup and equipment used for the analysis. Recommendations and guidelines for uncertainty quantification and errors sources in PIV measurements were presented by several authors [20] [19] [18]. [18] pointed out five specific categories and corresponding sources of errors, summarized in Table 1.

Table 1. Uncertainty and source of errors related to PIV measurements [18]

\begin{tabular}{ll} 
Category & Uncertainty and source of errors \\
\hline Calibration & $\begin{array}{l}\text { misalignment and/or distance between the laser } \\
\text { sheet and the calibration plate; image and } \\
\text { physical distance of calibration plate dots, lens } \\
\text { aberration and magnification, image distortion } \\
\text { caused by lens, and camera chip distortion }\end{array}$ \\
\hline Time Interval & pulse timing of both camera and laser \\
\hline $\begin{array}{l}\text { Displacement of } \\
\text { particle image }\end{array}$ & $\begin{array}{l}\text { mis-matching of paired particles image; camera } \\
\text { viewing angle, laser power fluctuation, sub-pixel } \\
\text { particle image movement }\end{array}$ \\
\hline Measurement & $\begin{array}{l}\text { uniformity of the particle distribution and origin } \\
\text { position }\end{array}$ \\
\hline Experiment: & particle lag and out-of-plane velocity component \\
\hline
\end{tabular}

As presented in Table 1, there are different sources of errors that can decrease the accuracy and reliability of the velocity vectors obtained by the PIV measurement. In this section, we decided to focus on the errors due to the installation and alignment since a misalignment between the light sheet plane and the flow direction can originate a velocity field that does not represent the flow behavior. Moreover, if the measurement plane does not coincide exactly with the plane selected for calibration, wrong results can be obtained [19].

\section{Laser and Camera Alignment}

The alignment between the CCD camera and the laser is crucial to minimize the measurement errors. In that sense, it is important to ensure a right angle between the position of the camera and the light sheet [19]. To ensure this perpendicularity between the laser and the camera throughout the experiments, a structure was designed and constructed. The 3D model performed using the SolidWorks ${ }^{\circledR}$ software is depicted in Fig. 2.

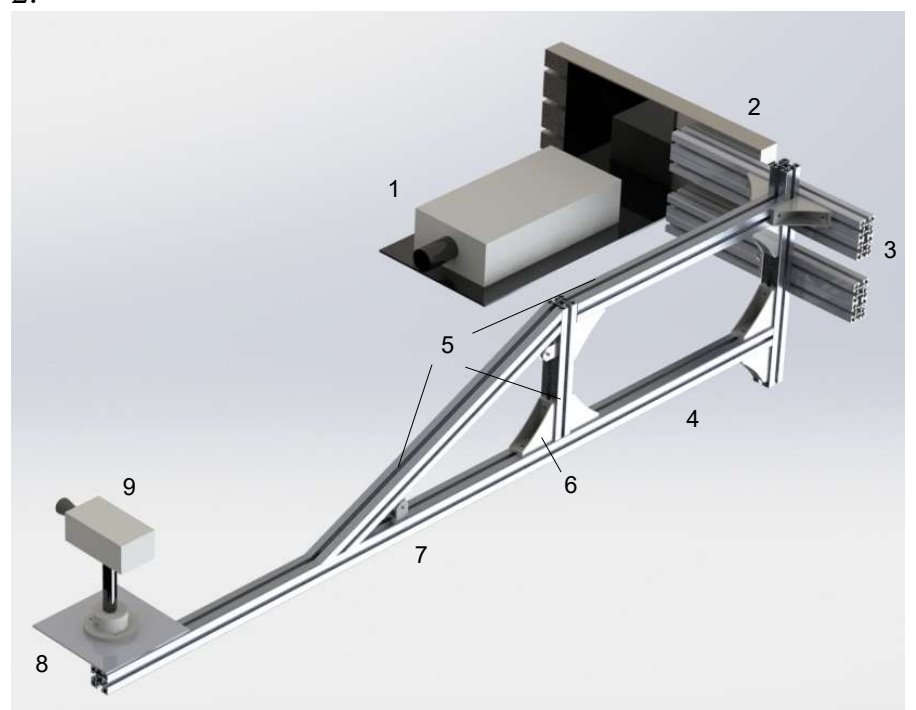

Fig. 2 - Structure for the alignment of the CCD camera and the laser.

The structure consists on a support for the laser (1) fixed on a platform (2) that ensure its vertical and horizontal motion. Two horizontal bars (3) fixed to the platform give the distance necessary between the camera (9) and the laser (specified by dimensions of the experimental setup) and follows the platform motion. A main aluminum bar (4) with the length that ensures the collection of the images inside the test chamber (through the glass door of the oven), supports the camera. A specific component (8) was designed and constructed by $3 \mathrm{D}$ printing to allow the fixing of the camera axis. This specific axis was preserved from the Manfrotto 290 xtra carbon tripod since it allows the positioning of the camera in three axes. Several aluminum bars (5), rectangular (6) and angular supports (7) were used to provide resistance to the structure.

The correct assembly of all these parts is fundamental since the horizontality and verticality of the structure must be ensured, as well as the right angle between the structure and the laser and camera. To guarantee the structure requirements, specific measurement instruments were used such as torpedo level, angle protractor (resolution $\pm 0.1^{\circ}$ ) and caliper (resolution $\pm 0.01 \mathrm{~mm}$ ). The structure was assembled to the oven, ensuring 
that the laser beam passes exactly in the middle of the oven window, being the images captured by the camera through the glass door. With this configuration, it is expected to capture the central row of nozzles with a thickness equivalent to the thickness of the laser sheet. Regarding this thickness, [19] mentioned that the minimum PIV sample size must be at least the same than the light sheet thickness. Moving the laser through the platform, the camera follows the movement with an error of $\pm 0.05 \mathrm{~mm}$ in both horizontal and vertical direction. Throughout the experiments, different measurement layers can be obtained and analyzed by moving the laser sheet. With the implementation of this structure, the errors of misalignment between the laser and the camera will be significantly reduced.

After the definition of the correct setting of the camera and the laser, their position must be fixed before starting the calibration. As mentioned by [19], to minimize errors, it is important to ensure that the calibration conditions are the same than the measurement conditions.

\section{Seeding Particles}

As mentioned previously, the seeding particles plays an important role in the accuracy of the PIV measurement. From [22], it is known that the tracer particles cannot affect the dynamic of the flow neither changing their properties during the measurement or interact with each other. Furthermore, they must be randomly and uniformly distributed across all the fluid with a specific concentration in order to increase the accuracy of the measurements. However, achieving an optimum flow seeding is the most difficult part of PIV experiments, a reduced concentration of particles will generate inconclusive velocity fields, while excessive seeding can generate medium opacity [15]. According to [15], if the working fluid is air, the seeding needs to be entrained in the air and then introduced upstream of the measurement region. However, the correct concentration of tracer particles is challenging, since in addition to ensure a uniform distribution of the seeding across the test chamber, the deposition of the particles on walls is another factor difficult to overcome. Two problems arise from this deposition: first the seeding system must insert more particles to compensate the ones that fixed at the walls; second, window deposition is also a limiting factor, since the transparency is reduced, leading to a deformation of the image captured that generates errors of measurement. This implies a continuous cleaning of both door and window of the experimental setup used in this study. This issue was already considered during the design of the oven, and thus, the quick and easy cleaning of the glass and door was ensured by a good access to these parts.

In addition to the precise distribution of the seeding throughout the test chamber, the selection of the seeding flow for the accurate tracking of the working flow is crucial to obtain accurate measurements. According to [23], a compromise between a reduced particle size to improve the flow tracking and a large particle size to improve the light scattering must be ensured. The same author suggested a scattering particle concentration of approximately 15 particles per interrogation area to obtain a velocity field with high accuracy.

Due to the importance of the selection of tracer particles that are suitable for the tracking of the flow in study and for the entire installation, a seeding characterization was performed and presented in the following section.

\section{Seeding characterization for flow visualization}

This study complements the research conducted by Barbosa et al. [24]. The authors mentioned that Shell Ondina EL oil used by the Aerotech Smoke Generator for smoke production was not appropriate for the flow tracking since the particles selected present a too high mean diameter, $14.3 \mu \mathrm{m}$. According to Melling [25], an acceptable tracking of turbulence or high speed gas flow is ensured by particles with a diameter of $1 \mu \mathrm{m}$ or smaller, however, a diameter up to $2-3 \mu \mathrm{m}$ seems to be acceptable for a moderate frequency response of $1 \mathrm{kHz}$.

In that sense, two fluids were selected to replace the Shell Ondina EL oil, a virgin olive oil and Johnson baby oil. To analyze if one or both oils complie with the requirements mentioned by [25] the same experiment presented by [24] was performed.

The oils were introduced separately in the Aerotech Smoke Generator, after proper cleaning, which generates smoke droplets. The size of the droplets was measured using a Laser Diffraction Technique, through a Malvern 2600, as depicted in Fig. 3. The particles are introduced into the analyzer beam formed by the low-power He-Ne laser, leading to the scattering of the laser light that is collected by a receiver lens. In its turn, the unscattered light is monitored in order to determine the volume concentration of the sample. A lens of $63 \mathrm{~mm}$ was selected for the measurement, since it allows a measurement range between $0.5 \mu \mathrm{m}$ and $100 \mu \mathrm{m}$.

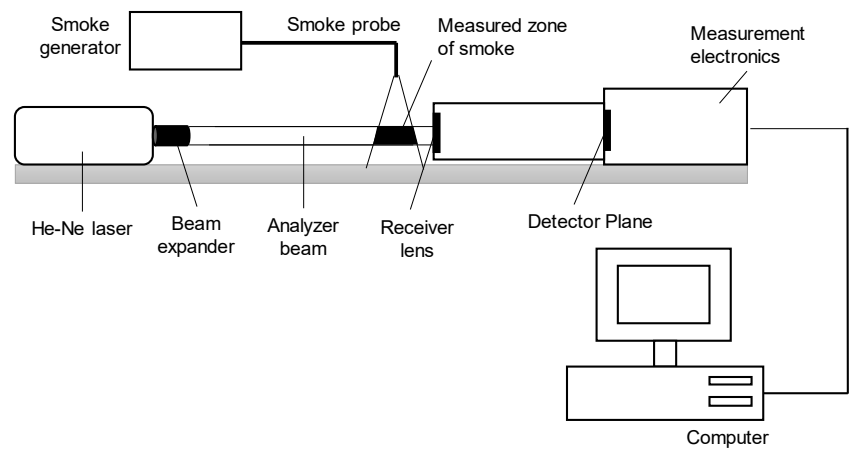

Fig. 3 - Laser Diffraction experiment [24].

The measurements performed by the Malvern 2600 are expressed in terms of volume distribution (Table 2). The software processes the data and express the results in terms of two mean diameters, De Brouckere and Sauter, that represent the volume or mass moment mean and the surface area moment mean respectively. These diameters are typically employed in particle size measurement since they specify around which central point of the frequency (surface area or volume/mass) the distribution varies [26]. 
Table 2 - Mean diameter measured by Malvern 2600.

\begin{tabular}{lcc}
\multirow{2}{*}{ Mean diameter } & \multicolumn{2}{c}{ Value $\pm \mathbf{0 . 0 1}[\boldsymbol{\mu m}]$} \\
\cline { 2 - 3 } & Olive oil & Johnson baby oil \\
\hline De Brouckere & 3.14 & 4.54 \\
\hline Sauter & 3.09 & 4.51 \\
\hline
\end{tabular}

Through the analysis of the results presented in Table 2, it seems that the most appropriate seeding is the virgin olive oil since the particles mean diameter is approximately $3 \mu \mathrm{m}$. This diameter complies with the requirement of an accurate flow tracking presented by [25]. Therefore, these tracer particles were selected for the PIV measurement.

To analyze if the seeding selected is appropriate for the flow visualization and PIV measurement using the experimental setup previously presented, the particles were introduced in several points (holes) over the oven and above the test chamber. Fig. 4 illustrates the experimental apparatus with the zones were the probe of the smoke generator was introduced, expressed by the red circles. The probe was introduced just before and after the centrifugal ventilator and above and below the honeycombs.

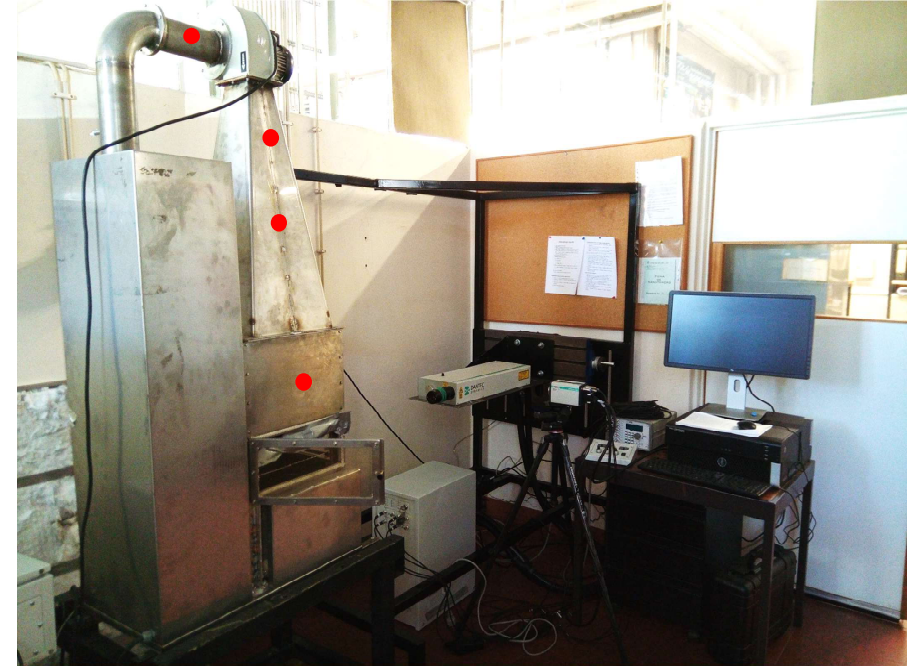

Fig. 4. Experimental apparatus and identification of the seeding zones.

The experiment was conducted to analyze the efficiency of the seeding and consists on the introduction of the smoke generator probe inside each zone indicated in Fig. 4. To minimize the deposition of the tracer particles on the oven walls, the air inside the setup was heated at a temperature of $120^{\circ} \mathrm{C}$. The probe was introduced when a constant temperature inside the oven was reached. While an experiment was performed in one specific region of the oven the other holes in study were closed with a high temperature aluminum tap in order to prevent leakage. However, the results obtained are not conclusive. No particles are observed in the test chamber when the ventilator is running in the case of the three points located farther from the test chamber. Some particles are observed when the probe is located just above the test section, nonetheless the flow is too irregular since the only element that separates the test chamber and the probe is the nozzle plate. In that sense, we decided to conduct a complementary study to try to understand which are the physical constraints that prevents the particle to flow into the test chamber.

\section{Experimental setup for seeding study}

The experimental setup for the study of the seeding consists of a ventilator (1), coupled to a flow regulator in order to control the velocity of the air flow throughout the experiment. The ventilator is linked to a diffuser (2) that directs the air to a stabilization chamber (3) in order to reduce the turbulence generated by the ventilator. An acrylic pipe is linked to the stabilization chamber, inside which a honeycomb structure (4) was placed, just at the exit of (3), to ensure the uniformization of the flow. A nozzle plate (5) with the same configuration between the nozzles than the one implemented in the oven was introduced inside the pipe. The PIV laser and camera were positioned perpendicularly at a distance that allows to capture the flow at the exit of the nozzles. The probe of the smoke generator is introduced after the honeycombs (red circular point in Fig 5) to allow an uniformization of the seeded flow throughout the pipe. In that sense, it is expected that the same quantity of seeded air flows through the nozzles.

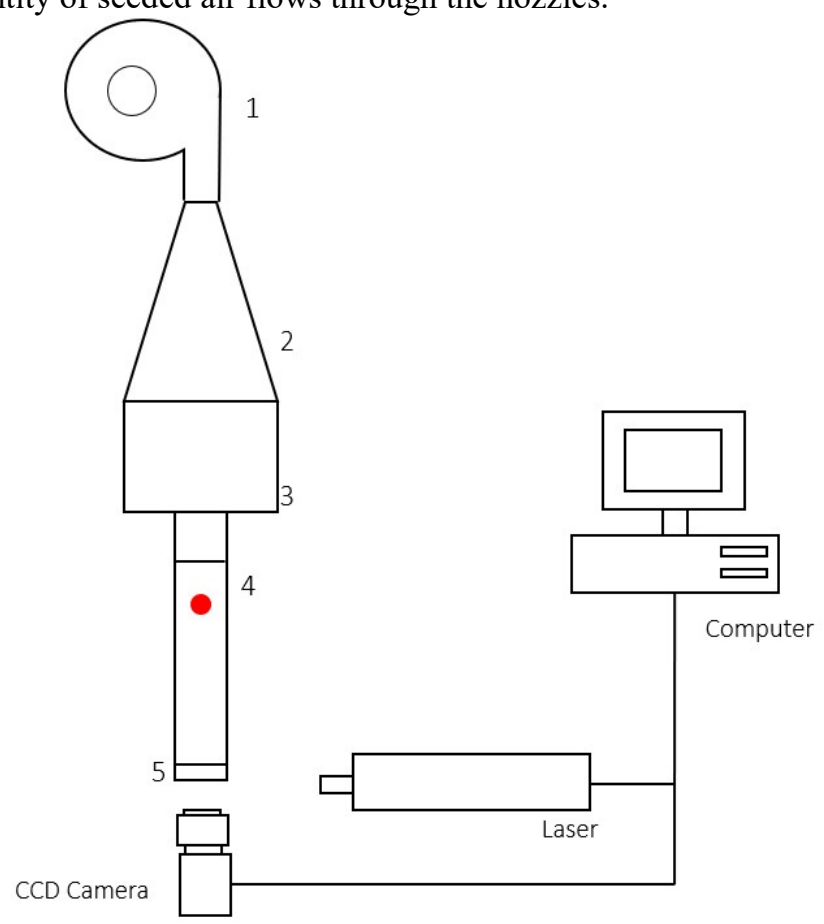

Fig. 5. Experimental apparatus for seeding study.

This experiment consists on the analysis of the jets flow profile, properly seeded, for different jet velocities. To perform this study, the frequency of the ventilator was changed from 5 to $20 \mathrm{~Hz}$, leading to the variation of the jet velocity at the exit of the nozzles. This velocity was measured using a Pitot tube connected to a digital manometer. The measuring equipment is 
depicted in Fig. 6 and the specifications presented in Table 3. The velocities were measured at the exit of the central nozzle and the values recorded are presented in Fig.7. As it can be observed, the velocity increases linearly with the increase of the ventilator frequency. A frequency of $5 \mathrm{~Hz}$ corresponds to a velocity of approximately $7 \mathrm{~m} / \mathrm{s}$ and $20 \mathrm{~Hz}$ to $27 \mathrm{~m} / \mathrm{s}$.

Table 3 - Specification of the manometer Love Controls.

\begin{tabular}{ccc}
$\begin{array}{c}\text { Range of } \\
\text { measurement }[\mathbf{P a}]\end{array}$ & $\begin{array}{c}\text { Air velocity } \\
\text { accuracy }\end{array}$ & $\begin{array}{c}\text { Resolution } \\
{[\mathbf{P a}]}\end{array}$ \\
\hline 0 to $2.5 \times 10^{3}$ & $\begin{array}{c} \pm 0.2 \% \text { full scale } \pm 1 \\
\text { digit }\end{array}$ & $1.0 \times 10^{-3}$ \\
\hline
\end{tabular}

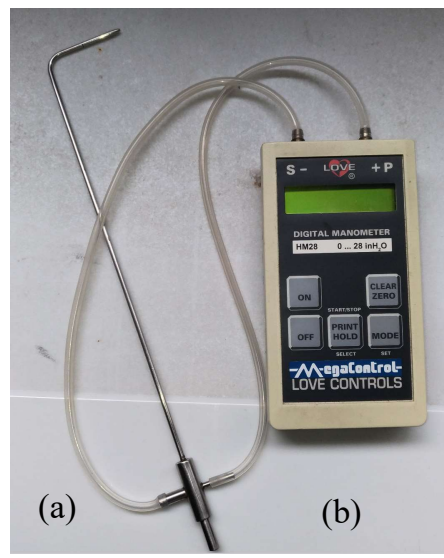

Fig. 6 - Devices for jet velocity measurement: (a) Pitot tube (b) Manometer.

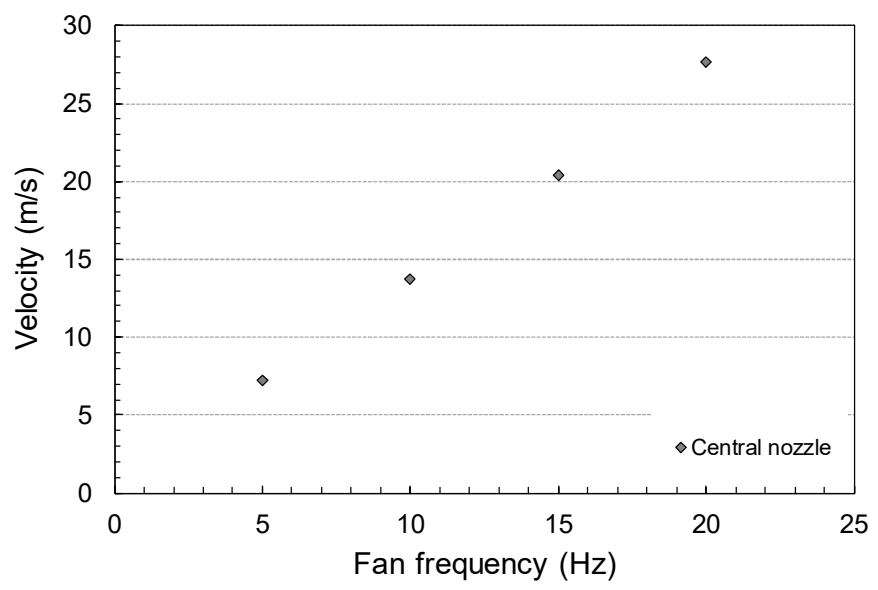

Fig.7 - Mean jet velocity variation with the fan frequency at ambient temperature

To avoid the deposition of the oil particles in the measurement area, we decided to perform the study without confinement, so the jets behave as free jets. The smoke generator probe debited the olive oil droplets inside the pipe at a flow rate of $2 \mathrm{~m}^{3} / \mathrm{h}$. The heater voltage of the smoke generator was fixed at $20 \mathrm{~V}$. The images captured by the CCD camera are presented in Fig. 8.

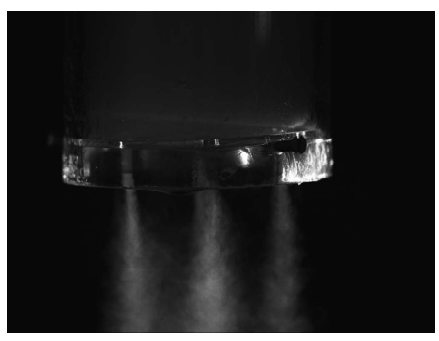

(a)

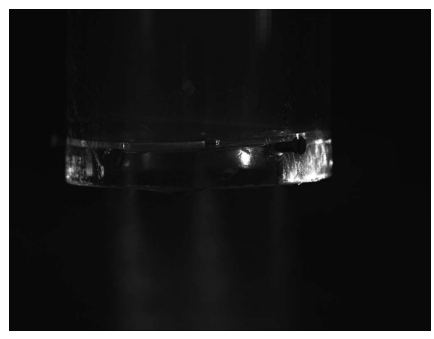

(d)

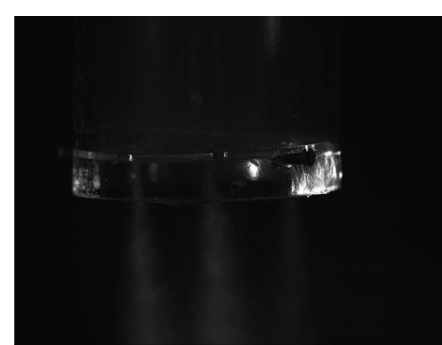

(b)

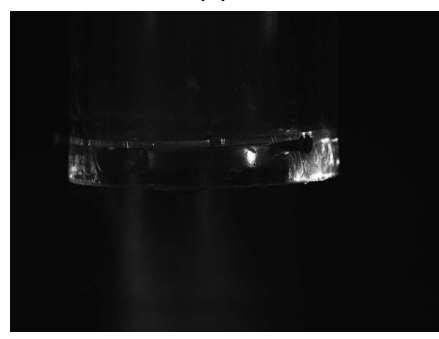

(c)
Fig. 8 - Jets flow behavior with the variation of the fan frequency (a) $5 \mathrm{~Hz}$ (b) $10 \mathrm{~Hz}$ (c) $15 \mathrm{~Hz}$ (d) $20 \mathrm{~Hz}$.

As it can be observed, the increase of the flow velocity leads to a decrease of the seeded flow. From this study it can be conclude that the increase of the velocity and consequently of the turbulence of the flow inside the pipe leads to a decrease of the particles that flows through the nozzles. The particles are trapped inside the vortices generated above the nozzle plate, being difficult for the camera to detect these particles and consequently, a reduction of the PIV efficiency occurs. Further studies must be conducted in order to determine the best conditions that allow the flow analysis for a higher range of velocities, since those that can actually be measured by the PIV system are low (at least around $7 \mathrm{~m} / \mathrm{s}$ ).

Looking at the data measured by the PIV system for case (a), presented in Fig.9, the scalar map vector of the velocity magnitude $\left(\sqrt{ } u^{2}+v^{2}\right)$ shows the velocity variation of the jets from the jet exit to the environment. The results demonstrate higher velocity values at the exit of the nozzle and along the jet axis, as it is expected. This velocity decreases with the increase of the distance from the nozzle exit at both $x$ and $y$ directions. Even if the jets develop in an unconfined space, some interaction can be observed, essentially between the central and left jets. This interaction leads to an increase of the turbulence and consequently of the flow velocity. Furthermore, a higher concentration of particles is identified in the central jet, showing that the seeding particles distribution over the working flow is not completely uniform. This non uniform distribution of the seeding particles can generate errors that affects the reliability and accuracy of the measurements performed by the PIV. 


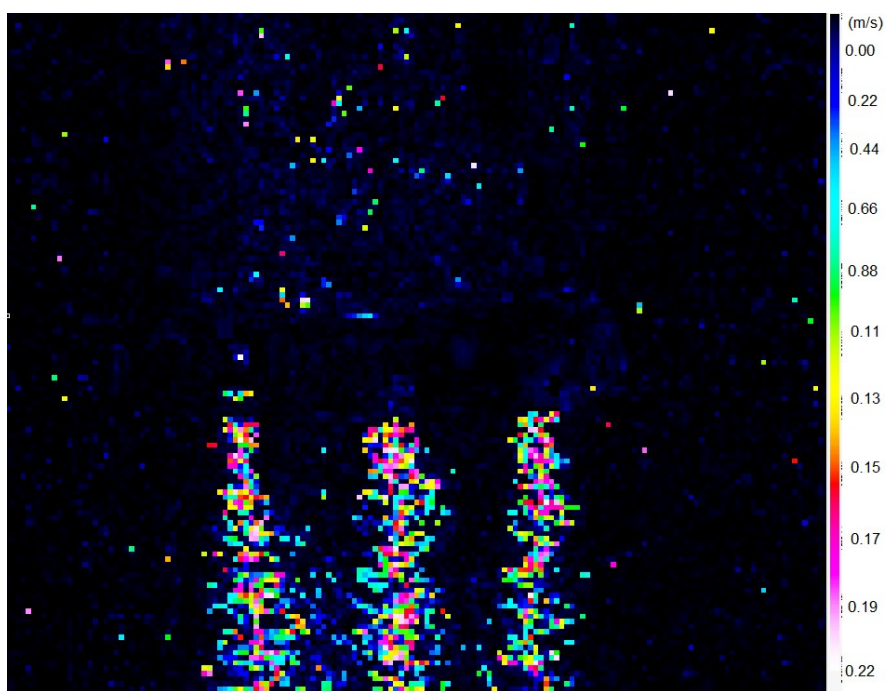

Fig. 9 - Scalar map of the velocity magnitude obtained by PIV measurement in case (a).

\section{Jets velocity}

In addition to the source of errors related to the PIV system, others related to the experimental setup per se must be identified and analyzed. One of the most important aspects related to the multiple jet impingement process and the success of its study is the uniformization of the flow throughout the test chamber. It is crucial to ensure that the air that flows through each nozzle is the same, that means, the air velocity at the nozzle exit must be the same, otherwise the conclusions obtained from the analysis of the jet's interactions can be wrong. In that sense, the measurement of the flow velocity inside the test chamber was conducted using the Pitot tube connected to the digital manometer previously presented. Since the nozzle plate contains more than 200 nozzles, just few of them were selected for this study: the central nozzle, two nozzles forward, two nozzles behind, one nozzle to the left of the central and one to the right. This configuration was selected due to the restricted motion of the Pitot tube since the test chamber is a confined space.

The experimental setup runs for specific conditions of velocity, given by the centrifugal fan and temperatures, controlled by the heaters. To understand the behavior of the system with the variation of these two parameters the study performed consists on the variation of the fan flow rate for two different air temperatures, $120^{\circ} \mathrm{C}$ and $150^{\circ} \mathrm{C}$, followed by the measurement of the velocity at the nozzles exit. These temperatures were selected for the multiple jet impingement study, it is why they must be analyzed in this section. The results obtained are presented in Fig. 10 and Fig. 11 respectively. The velocities obtained represents the mean of 10 values recorded by the pitot tube.

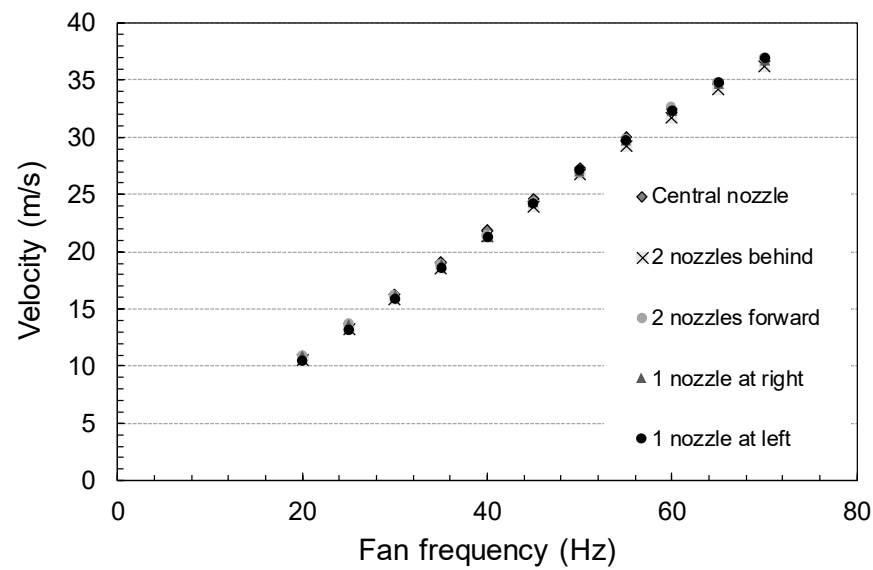

Fig. 10 - Mean jet velocity variation with the fan frequency at $\mathrm{T}=120^{\circ} \mathrm{C}$

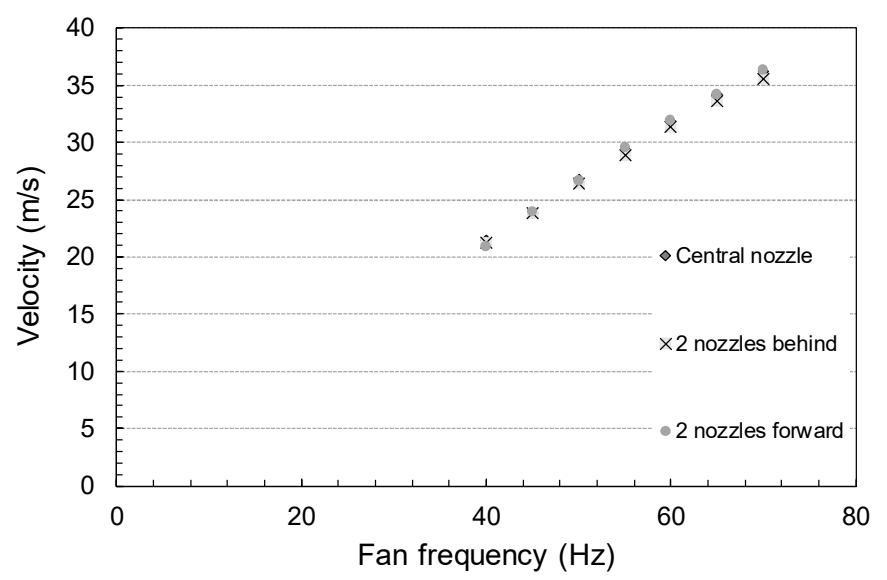

Fig. 11 - Mean jet velocity variation with the fan frequency at $\mathrm{T}=150^{\circ} \mathrm{C}$

As it can be observed, the velocity at the nozzle exit varies from $10 \mathrm{~m} / \mathrm{s}$ to approximately $37 \mathrm{~m} / \mathrm{s}$ and this velocity varies linearly with the increase of the fan frequency. Furthermore, the results show a slight discrepancy between the velocities measures in each nozzle increasing with the increase of the velocity. This demonstrates that at the center of the test chamber, the velocity at the nozzle exit is approximately uniform.

Focusing on the influence of the jet temperature on the jet velocity, the differences observed in both cases is very smooth. With a temperature of $120^{\circ} \mathrm{C}$ the velocity at the nozzle exit is approximately $1 \mathrm{~m} / \mathrm{s}$ higher than $150^{\circ} \mathrm{C}$. Moreover, using this last temperature, the limit of the system is reached at a frequency of $40 \mathrm{~Hz}$, i.e. to maintain a constant temperature of $150^{\circ} \mathrm{C}$ inside the test chamber, the fan must blow the air at least at flow rate equivalent to the $40 \mathrm{~Hz}$, otherwise the velocity inside the test chamber increases to higher values. That means that if an air jet temperature of $150^{\circ} \mathrm{C}$ is required, it is necessary to work with a jet velocity of about $20 \mathrm{~m} / \mathrm{s}$. 


\section{CONCLUSIONS}

This paper presents an experimental apparatus specially built for the study of multiple jet impingement process. In addition to the identification of the different components that compose the installation, the sources of errors are identified and analyzed. The study performed shows the complexity related to the PIV system and the limitations of the experimental setup. The sources of errors that influence the reliability and accuracy of the velocity field measurements are approached, showing that the seeding is one of the most complex factors related to the PIV measurements. The uniformization of the tracer particles distribution throughout the flow is difficult to obtain and the dust generated by the deposition of the seeding on the setup walls leads to a decrease of the results accuracy. The results demonstrate that the nozzle plate configuration is a limitation of the system, since the open area is largely reduced, generating high turbulence upstream the nozzles. To overcome the source of errors approached in this study, a structure to correct the misalignment between the laser and the camera was designed. Moreover, an experimental setup for the specific study of the seeding particles was developed and presented.

Future works will be performed in order to quantify the uncertainty related to the source of errors identified in this paper. The experiments regarding the seeding will proceed in order to obtain a solution that ensure accurate and reliable velocity measurements of multiple jet impingement in a confined space.

\section{ACKNOWLEDGMENTS}

The first author would like to express her gratitude for the support given by the Portuguese Foundation for Science and Technology (FCT) through the Research Grant PD/BD/128216/2016. This work has been supported by FCT within the Project Scope UID/CEC/00319/2019 (ALGORITMI) and Project Scope UID/EMS/04077/2019 (METRICS).

\section{NOMENCLATURE}

Temperature

$t \quad$ Time

$H \quad$ Nozzle to plate distance

(s)

Jet diameter

(m)

D

$\mathrm{S}$

u

$\mathrm{V}$

$v$

$x_{p}$

CCD

LDV Laser Doppler Velocimetry

Re Reynolds number

PIV Particle Image Velocimetry

S-PIV stereoscopic particle image velocimetry
TLC Thermosensitive or Transient Liquid Crystal

\section{REFERENCES}

[1] N. Zuckerman and N. Lior, "Jet Impingement Heat Transfer: Physics , Correlations, and Numerical Modeling," Adv. Heat Transf., vol. 39, no. 06, pp. 565631, 2006.

[2] A. Dewan, R. Dutta, and B. Srinivasan, "Recent trends in computation of turbulent jet impingement heat transfer," Heat Transf. Eng., vol. 33, no. 4-5, pp. 447460, 2012.

[3] V. Katti and S. V Prabhu, "Experimental study and theoretical analysis of local heat transfer distribution between smooth flat surface and impinging air jet from a circular straight pipe nozzle," Int. J. Heat Mass Transf., vol. 51, pp. 4480-4495, 2008.

[4] M. Angioletti, R. M. Di Tommaso, E. Nino, and G. Ruocco, "Simultaneous visualization of flow field and evaluation of local heat transfer by transitional impinging jets," Int. J. Heat Mass Transf., vol. 46, pp. 1703-1713, 2003.

[5] R. D. Keane and R. J. Adrian, "Optimization of particle image velocimeters . I . Double pulsed systems," Measrement Sci. Technol., vol. 1, pp. 1202-1215, 1990.

[6] U. Ullum, J. J. Schmidt, and P. S. Larsen, "Statistical analysis and accuracy of PIV data," J. Vis., vol. 1, no. 2, pp. 205-216, 1998.

[7] Y. Ichikawa, M. Motosuke, Y. Kameya, M. Yamamoto, and S. Honami, "Three-dimensional flow characterization of a square array of multiple circular impinging jets using stereoscopic PIV and heat transfer relation," J. Vis., vol. 19, no. 1, pp. 89-101, 2016.

[8] Y. Xing, S. Spring, and B. Weigand, "Experimental and Numerical Investigation of Heat Transfer Characteristics of Inline and Staggered Arrays of Impinging Jets," J. Heat Transfer, vol. 132, no. 9, p. 092201, 2010.

9] S. J. Kline and F. A. McClintock, "Describing uncertainties in single-sample experiments," Mech. Eng., vol. 75, no. 1, pp. 3-8, 1953.

[10] W. Li, M. Xu, J. Ren, and H. Jiang, "Experimental Investigation of Local and Average Heat Transfer Coefficients Under an Inline Impinging Jet Array, Including Jets With Low Impingement Distance and Inclined Angle," J. Heat Transf. Asme, vol. 139, no. 1, p. 12201, 2017.

[11] R. J. Moffat, "Describing the Uncertainties in Experimental Results," pp. 3-17, 1988.

[12] P. Grenson, O. Léon, P. Reulet, and B. Aupoix, "Investigation of an impinging heated jet for a small nozzle-to-plate distance and high Reynolds number: An extensive experimental approach," Int. J. Heat Mass Transf., vol. 102, pp. 801-815, 2016.

[13] A. Terzis, "On the correspondence between flow 
structures and convective heat transfer augmentation for multiple jet impingement," Exp. Fluids, vol. 57, no. 9, pp. 1-14, 2016.

[14] R. Ben Kalifa, S. Habli, N. M. Saïd, H. Bournot, and G. Le Palec, "Parametric analysis of a round jet impingement on a heated plate," Int. J. Heat Fluid Flow, vol. 57, pp. 11-23, 2016.

[15] C. Tropea, A. L. Yarin, and J. F. Foss, Springer Handbook of Experimental Fluid Mechanics. Springer Berlin Heidelberg, 2007.

[16] J. Westerweel, "Fundamentals of digital particle image velocimetry," Meas. Sci. Technol., vol. 8, pp. 13791392, 1997.

[17] J. Westerweel, "Fundamentals of digital particle image velocimetry," Meas. Sci. Technol., vol. 8, no. 12, pp. 1379-1392, 1997.

[18] P. Sabharwall, T. Conder, R. Skifton, C. Stoots, and E. S. Kim, "PIV Uncertainty Methodologies for CFD Code Validation at the MIR Facility," Idaho, 2013.

[19] M. Raffel, C. E. Willert, F. Scarano, and C. J. Kähler, Particle Image Velocimetry, Third ed. Switzerland: Springer, 2018.

[20] A. Sciacchitano, D. R. Neal, B. L. Smith, A. Sciacchitano, B. Wieneke, and F. Scarano, "PIV uncertainty quantification by image," 2013.

[21] K. T. Christensen and F. Scarano, "Uncertainty quantification in particle image velocimetry," Measrement Sci. Technol., vol. 26, 2015.

[22] a Melling, "Tracer particles and seeding for particle image velocimetry," Meas. Sci. Technol., vol. 8, no. 12, pp. 1406-1416, 1997.

[23] I. Grant, "Particle image velocimetry: a review," Proc. Inst. Mech. Eng., vol. 211, no. C, pp. 55-76, 1997.

[24] Barbosa, FV; Silva, JPV; Ribeiro, PE; Teixeira, SFC; Soares, DF; Santos, D; Cerqueira, MF and Teixeira, JCF (2018). An Experimental Setup for Multiple Air Jet Impingement Over a Surface. In ASME (Ed.), Volume 8B: Heat Transfer and Thermal Engineering; paper No. IMECE2018-87995; doi:10.1115/IMECE2018-87995.

[25] A. Melling, "Tracer particles and seeding for particle image velocimetry," Meas. Sci. Technol., vol. 8, pp. 1406-1416, 1997.

[26] A. Rawle, "Basic principles of particle size analysis," UK, 2017. 